\title{
RTT Radiographic surveillance of minimally and moderately complex renal cysts
}

\author{
Ahmed H. Gabr, Yehoshua Gdor, William W. Roberts and J. Stuart Wolf Jr \\ Department of Urology, University of Michigan Health System, Ann Arbor, MI, USA \\ Accepted for publication 8 August 2008
}

Study Type - Prognosis (case series) Level of Evidence 4

\section{OBJECTIVE}

To assess the effectiveness of radiographic surveillance for managing minimally and moderately complex renal cysts.

\section{PATIENTS AND METHODS}

Forty-three patients with 50 minimally or moderately complex renal cysts underwent radiographic surveillance at our institution. Study inclusion criteria were surveillance for $>2$ years ( 36 patients, mean follow-up 3.0 years) or surveillance for $>6$ months with subsequent surgical excision (seven patients, mean follow-up 3.3 years).

\section{RESULTS}

The complexity of the renal cysts was in the form of high attenuation before contrastenhanced imaging ('hyperdense') in 29 patients, thin septations in nine, borderline enhancement in six, thin calcifications in five, and a thick wall in one. The mean initial largest dimension was $2.9 \mathrm{~cm}$ and the mean final dimension was $3.0 \mathrm{~cm}$, with the size increased in 29 cysts, decreased in 14 and with no change in seven. The cyst character worsened in seven patients, improved in four and did not change in 39. Eventually seven patients had surgery (laparoscopic partial nephrectomy in five and laparoscopic radical nephrectomy in two), which revealed renal cancer in five. Surgical intervention was prompted by growth alone in two patients, growth and worsening of cyst characteristics in two, new onset of flank pain in one, and appearance of an enhancing nodule in the wall or septa in two.

\section{CONCLUSION}

Radiographic surveillance is an effective method for managing patients with minimally or moderately complex renal cysts. Malignant lesions can be identified and removed while still of low grade and contained, and surgery can be avoided in most patients.

\section{KEYWORDS}

kidney diseases, cystic, carcinoma, renal cell, tomography, X-ray, MRI

\section{INTRODUCTION}

More frequent cross-sectional imaging had led to an increase in the detection of incidental renal lesions. Although simple cysts comprise most of these, the number of patients with more complicated lesions is nonetheless considerable. Differentiating complex cystic lesions of the kidney that need surgical intervention from those that do not is a difficult and common diagnostic problem. Bosniak [1] described a classification system that assists in making this distinction. Some cysts can be easily diagnosed as benign while others are very complex and are usually malignant, and therefore are best managed surgically. Many cystic lesions are minimally or moderately complex, but with a notinsignificant chance of malignancy. The typical management is to follow such lesions radiographically, with two assumptions. The first assumption is that if the lesion grows significantly or worsens in character, then the likelihood of malignancy is greater, and therefore surgery is more likely to be beneficial. The second assumption is that if the lesion does not grow or worsen in character over a period (3-5 years) then the chance of underlying malignancy that would harm the patient is so low that further followup is not required.

These assumptions underlying the management strategy of radiographic surveillance have been validated in published series with 38-42 patients, and a mean follow-up of 2.6-5.8 years $[2,3]$. The objective of the present study was to evaluate the effectiveness of urologistdirected radiographic surveillance in the management of minimally to moderately complex renal cysts.

\section{PATIENTS AND METHODS}

All 43 patients, with 50 minimally or moderately complex renal cysts (seven had two cysts), who entered radiographic surveillance rather than surgical intervention in the period July 2004 to August 2007 at our institution, were identified from chart review and assessed retrospectively.

The study group consisted of 24 men and 19 women (mean age 63.3 years, range $25-85$ ). All patients underwent surveillance in the form of cross-sectional imaging (CT or MRI) at 6 and 12 months after initial cross-sectional imaging, and then annually for 3-5 years. All imaging studies included series without and then with i.v. contrast material; for CT we used 2.5-5 mm slice thicknesses. The complexity of lesions was assessed on the basis of the presence of high pre-contrast attenuation ('hyperdense'), septations, calcification, wall thickness or irregularity, or contrast enhancement. According to the categorization of Bosniak [1,4-7], all were category II ('minimally complex') or IIF ('moderately complex'). More complex lesions (i.e. Bosniak category III) were not included in this analysis, nor were the few Bosniak category II lesions for which primary surgical intervention was chosen. All 
FIG. 1. $a, 2.7 \mathrm{~cm}$ left upper pole renal cyst with calcifications on CT; $b, 3.1$ years later, the lesion is stable in size and characteristics.
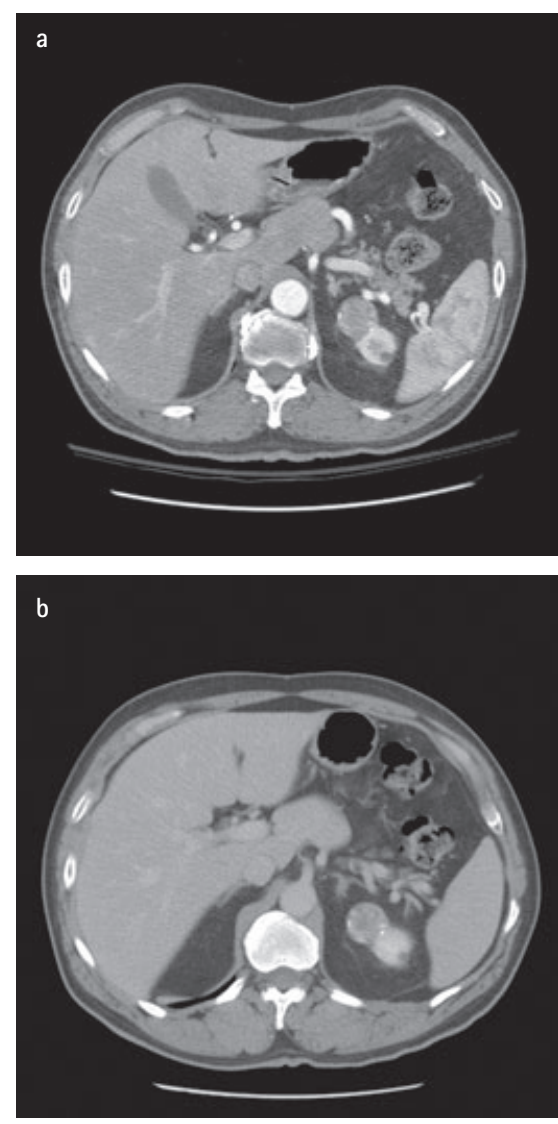

preoperative management, including the review of radiographic imaging, was directed by one urologist (J.S.W.).

Patients included in the study had initially presented with renal cyst(s) that had, on CT or MRI (without and with i.v. contrast material): hyperdensity, thin septations, borderline enhancement of otherwise thin walls or septa, thick walls or septa with no enhancement, or thin calcifications (e.g. Bosniak category II or IIF complex renal cysts). Patients with more worrisome characteristics were excluded. Also, patients were included only if they had: (i) radiographic surveillance for $>2$ years (36, mean follow-up of 3.0 years); or (ii) radiographic surveillance for $>6$ months with subsequent surgical excision (seven, mean follow-up 3.3 years).

\section{RESULTS}

The 43 patients with 50 minimally or moderately complex renal cystic lesions were
FIG. 2. $a, 3.4 \mathrm{~cm}$ right posterior renal cyst with multiple septations on MRl; $\boldsymbol{b}$, Growth to $3.9 \mathrm{~cm}$ 1 year later prompted laparoscopic partial nephrectomy, which revealed a Fuhrman grade 2 clear cell RCC, pT1, with negative surgical margins.
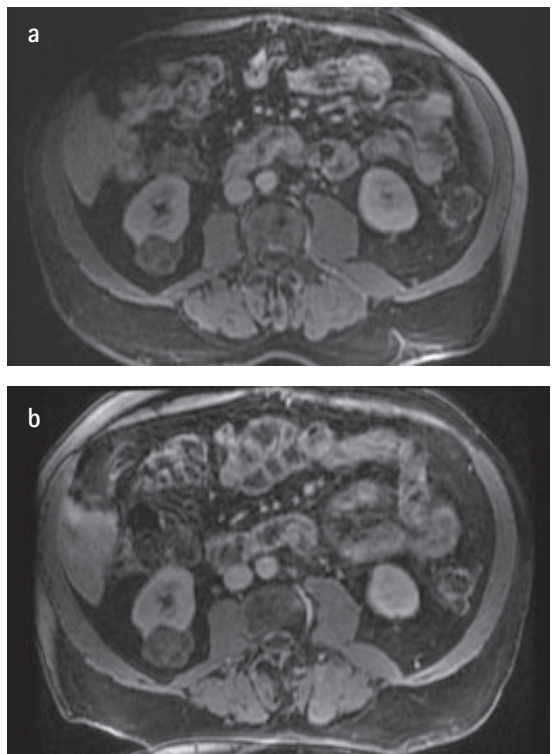

followed from December 2001 to September 2007, with a mean (range) follow-up of $3.1(0.5-8)$ years. The mean initial largest dimension was $2.9(0.8-8.7) \mathrm{cm}$ and the mean final dimension was $3.0(1.0-9.4) \mathrm{cm}$. Over the course of surveillance, the size increased in 29 cysts ( 24 by $<1 \mathrm{~cm}$ and five by $>1 \mathrm{~cm}$, mean 1.1), decreased in 14 ( 13 by $<1 \mathrm{~cm}$ and one by $>1 \mathrm{~cm}$, mean $-1.3 \mathrm{~cm}$ ), and did not change in seven (Figs 1,2). Reporting the most prominent initial cyst characteristic, the complexity of the renal cysts was in the form of hyperdensity in 29 , septations in nine, borderline enhancement of otherwise thin walls or septa in six, thin calcification(s) in five and a thick non-enhancing wall in one. Over the course of surveillance, the character of the renal lesion worsened in seven patients, improved in four and did not change in 39 (Table 1).

Seven of 43 patients had surgery, in the form of laparoscopic partial nephrectomy in five and laparoscopic radical nephrectomy in two. Surgery was prompted by growth with no change in character in two patients (pathology revealed a complex benign cyst in one with $2 \mathrm{~cm}$ of growth over 2 years, and malignancy in one, with $5 \mathrm{~mm}$ of growth over 1 year), growth and worsening of cyst characters in two (increasing heterogeneity of a hyperdense interior in one, with $8 \mathrm{~mm}$ of growth over 2.9 years, and development of a thick enhancing wall in one, with $3.8 \mathrm{~cm}$ of growth over 8 years; pathology showed renal cancer in both), new onset flank pain in one (pathology showed a benign renal cyst), and appearance of an enhancing nodule in the wall or septa in two (renal cancer confirmed in both). Of these seven lesions, three were minimally complex (i.e. the only abnormalities were homogenous hyperdensity, one or two fine septa, or one fine calcification) and four were moderately complex. Malignancy was found in one of the minimally complex and all four of the moderately complex lesions. Of the malignancies, three were Fuhrman grade 1 or 2 clear cell RCCs, two were papillary RCCs, and all were pathological stage T1. Among the six patients with growth or worsening of characteristics that prompted surgery, the mean growth was $1.0 \mathrm{~cm}$ over a mean follow-up of 2.9 years. Among the three patients with worsened cyst characteristics who continue on surveillance, the wall has thickened but the cyst has shrunk in one, there has been a slight increase in homogenous hyperdensity in one, and there has been the development of a faint calcification in one. Because there was no growth of the three lesions, continued surveillance has been selected.

Among the 36 patients with 43 cysts that did not require surgery (one of the surgically treated patients has a contralateral cyst that is still undergoing surveillance), 13 with 16 cysts have completed a 3-5-year surveillance regimen and have been discharged from clinic (mean follow-up 3.6 years), while 23 with 27 cysts are still under surveillance (mean follow-up 2.7 years). Among these 43 cysts, lesion size has increased in 24, decreased in 12 , and remained stable in seven, with an overall mean growth of $0.04 \mathrm{~cm}$.

\section{DISCUSSION}

In 1986, Bosniak [1] proposed a classification system for cystic renal masses, and elaborated further in later publications [4-7]. Category I lesions are simple cortical cysts; category Il lesions are minimally to moderately complicated cysts for which radiographic surveillance is usually recommended, as the risk of malignancy is 5-10\% [8-12]. Bosniak distinguished between minimally complex lesion that do not require surveillance (category II) and moderately complex lesions 
TABLE 1 Changes in the character of renal cysts

\begin{tabular}{|c|c|c|c|c|c|c|c|}
\hline \multirow[b]{2}{*}{ Initial character } & \multicolumn{7}{|c|}{ Final character } \\
\hline & Calcification & Enhancement & Hyperdense & Septations & Simple Cyst & Thick wall* & Total \\
\hline Calcification & 4 & 0 & 0 & 0 & 1 & 0 & 5 \\
\hline Enhancement & 0 & 4 & 0 & 0 & 1 & 1 & 6 \\
\hline Hyperdense & 1 & 0 & 25 & 0 & 2 & 1 & 29 \\
\hline Septations & 0 & 0 & 0 & 8 & 0 & 1 & 9 \\
\hline Thick wall & 0 & 0 & 0 & 0 & 0 & 1 & 1 \\
\hline
\end{tabular}

*The two cysts that developed wall nodules are included as wall thickening.

that need to be followed (category IIF). In our experience, the distinction between II and IIF is subjective and difficult to define, so we have elected to follow all category II lesions. This policy appears to be justified, in that one of the four confirmed malignancies in the present series was in a lesion that initially was a minimally complex renal cyst. $(1.3 \mathrm{~cm}$ homogenous, spherical, and hyperdense cyst that was stable for over 2 years until it grew and became heterogeneous). Category III lesions are the truly 'indeterminate' cystic masses that are as likely to be benign as malignant; surgery is recommended in medically suitable candidates. Category IV lesions are essentially solid lesions with cystic components, and are nearly always malignant.

The most difficult distinction is also the most important, i.e. between Bosniak categories II and III. The Bosniak system is useful in helping to categorise cystic renal lesions, although variability in its application and results have been reported [8-12]. At our institution we are following some medically unfit patients with Bosniak category III and IV cystic renal masses (and some patients with solid lesions [13]), but these patients were not included in the current study.

If the lesion grows slightly and slowly, this change is not necessarily worrisome as long as the cyst characteristics remain unchanged. However, significantly worsening cyst characteristics suggest malignancy and should prompt surgical exploration. To our knowledge, no guidelines have been offered for an absolute growth threshold that warrants intervention for observed masses, in the absence of worsening cyst character. In the present series we have some patients with slow-growing cysts who we are continuing to follow, as long as there are no changes in lesion characteristics, if they are relatively unfit for surgery. Our threshold for intervention is lower if the initial characteristics are at the worse end of the spectrum, and in younger and healthier patients. In general, we recommend intervention if there is sustained growth of $>5 \mathrm{~mm}$ per year, but the clinical condition of the patient is a critical factor in the decisionmaking process. In the present series, nine patients had growth of $>5 \mathrm{~mm}$ over the course of surveillance, of whom three had surgery. Of these three, two had growth of $>5 \mathrm{~mm}$ per year and one of $<5 \mathrm{~mm}$ per year but also had worsening characteristics. Among the other six patients, the annual growth rate was $<5 \mathrm{~mm}$. If surgery is elected, cyst exploration (with partial or radical nephrectomy depending on findings) appears to be a reasonable choice [14].

Certainly surgical intervention is not appropriate as the routine initial treatment of Bosniak II cystic renal masses, as only five of 50 renal lesions in the present series were confirmed to be malignant. This $10 \%$ incidence of malignancy is at the upper range of that reported previously, but our selection criteria for this study were such that the surgical group is over-represented relative to those patients not undergoing surgery. As radiographic surveillance for complex renal cysts is limited in duration, it is not as onerous as the lifelong regimen for small solid renal masses. As such, radiographic surveillance is not limited to medically unfit individuals, as in the case of solid renal masses. Nonetheless, some patients might prefer surgical intervention rather that being tied to the follow-up schedule. If surveillance is elected, we recommend cross-sectional imaging without and then with i.v. contrast agent 6 and 12 months after the initial examination, and then annually for $3-5$ years. Slow growth, with no change in cyst characteristics, might necessitate a longer follow-up. Moreover, even in the absence of cyst growth the follow-up duration depends on many factors, including size of the lesion and its complexity, in addition to the patient's medical condition. While a 3-5-year follow-up might be sufficient in older patients in the absence of cyst growth, in younger patients a longer follow-up might be appropriate.

Our selection criteria for surgery appear to be reasonable, in that five of the six patients who had surgery for indications other than pain also had cancer. What is unknown, of course, is how many of the patients with no surgery harbour an occult renal cancer. In the present series, pathological data were available in only seven patients who had surgical exploration, and in the rest we inferred benign lesions from the radiographic information. The duration of follow-up in the present series is reassuring in this regard.

In summary, radiographic surveillance is an effective method for managing patients with minimally and moderately complex renal cysts. Malignant lesions can be identified and removed while still low-grade and contained, and surgery can be avoided in most patients.

\section{CONFLICT OF INTEREST}

None declared.

\section{REFERENCES}

1 Bosniak MA. The current radiological approach to renal cysts. Radiology 1986; 158: $1-10$

2 Israel CM, Bosniak MA. Follow-up of moderately complex cystic lesions of the kidney (Bosniak category IIF). J Uro/ 2003; 181: 627-33 
3 Curry NS, Cochran ST, Bissada NK. Cystic renal masses: accurate Bosniak classification requires adequate renal CT. AJR Am J Roentgenol 2000; 175: 33942

4 Bosniak MA. Difficulties in classifying cystic lesion of the kidney. Urol Radiol 1991; 13: 91-3

5 Bosniak MA. Problems in the radiologic diagnosis of renal parenchymal tumors. Urol Clin North Am 1993; 20: 217-30

6 Bosniak MA. Diagnosis and management of patients with complicated cystic lesions of the kidney. AJR Am J Roentgenol 1997; 169: 819-21

7 Bosniak MA. The use of the Bosniak classification system for renal cysts and cystic tumors. J Urol 1997; 157: 1852-3
8 Aronson S, Frazier HA, Baluch JD, Hartman DS, Christenson PJ. Cystic renal masses: usefulness of the Bosniak classification. Urol Radiol 1991; 13: 8390

9 Brown WC, Amis ES Jr, Kaplan SA, Blaivas JG, Axelrod SL. Renal cystic lesions: predictive value of preoperative computerized tomography. J Urol 1989; 141: 426A

10 Wilson TE, Doelle EA, Cohan RH, Wojno $\mathrm{K}$, Korobkin M. Cystic renal masses: a reevaluation of the usefulness of the Bosniak classification system. Acad Radiol 1996; 3: 564-70

11 Cloix P, Martin X, Pangaud C et al. Surgical management of complex renal cysts: a series of 32 cases. J Urol 1996; 156: $28-30$
12 Siegel CL, McFarland EG, Brink JA, Fisher AJ, Humphrey P, Heiken JP. CT of cystic renal masses: analysis of diagnostic performance and interobserver variation. AJR Am J Roentgenol 1997; 169: 813-8

13 Siu W, Hafez KS, Johnston WK III, Wolf JS Jr. Growth rates of renal cell carcinoma and oncocytoma under surveillance are similar. Urologic Oncol 2007; 25: 115-9

14 Bellman GC, Yamaguchi R, Kaswick J. Laparoscopic evaluation of indeterminate renal cysts. Urology 1995; 45: 106670

Correspondence: J. Stuart Wolf Jr, Department of Urology, University of Michigan, 3875

Taubman Center, 1500 East Medical Center Dr, Ann Arbor, MI 48109-0330, USA.

e-mail:wolfs@umich.edu 